\title{
The Role of CETP Inhibition in Dyslipidemia
}

\author{
Karim El Harchaoui, MD, Wim A. van der Steeg, MD, \\ Erik S.G. Stroes, $M D, P h D$, and John J.P. Kastelein, $M D, P h D$
}

\section{Corresponding author \\ Karim El Harchaoui, MD \\ Department of Vascular Medicine, Academic Medical Centre, \\ University of Amsterdam, Room F4-159.2, Meibergdreef 9, \\ Postbus 22660, 1105 AZ Amsterdam, The Netherlands. \\ E-mail: a.el-harchaoui@amc.uva.nl}

Current Atherosclerosis Reports 2007, 9:125-133

Current Medicine Group LLC ISSN 1523-3804

Copyright ( 2007 by Current Medicine Group LLC

Cholesteryl ester transfer protein (CETP) inhibitors are currently being investigated because of their ability to increase high-density lipoprotein cholesterol levels. In various metabolic settings, the relationship between CETP and lipoprotein metabolism is complex and may depend largely on the concentration of triglyceriderich lipoproteins. Two CETP inhibitors, JTT-705 and torcetrapib, are in an advanced phase of development. Following hopeful intermediate results, a large endpoint study using torcetrapib has just been discontinued due to increased mortality in torcetrapib-treated subjects. In this review we summarize clinical data on the use of CETP inhibitors.

\section{Introduction}

Since reports in the late 1980s linking cholesteryl ester transfer protein (CETP) deficiency to high-density lipoprotein cholesterol (HDL-C) elevation, strong interest in developing drugs that can inhibit CETP has arisen. At this moment, at least six CETP inhibitors are being evaluated [1], two of which have reached phase III clinical trials. The potency of CETP inhibition has been underscored by HDL-C increases of up to $100 \%$, which clearly exceeds the efficacy of currently available HDL-C-raising therapies. In comparison, nicotinic acid, which is at present the most powerful drug to increase HDL-C, can raise HDL$\mathrm{C}$ levels by $30 \%$ at most [2]. The unanswered question remains whether a pharmacologic increase in HDL-C will result in a decrease in coronary artery disease (CAD). In support of this idea, HDL-C has consistently been found to be inversely related to the risk of CAD in several large, prospective population studies. In fact, several studies support the effectiveness of nicotinic acid-associated
HDL-C for reducing CAD. However, the atherogenic effects of CETP are still being debated. Very few studies have actually studied the relationship between CETP and CAD risk in humans. The first prospective study suggesting a relationship between CETP deficiency and CAD was the Honolulu Heart Study, although these results were mitigated in a follow-up study [3]. In a large prospective study, CETP concentration was a positive determinant of CAD, but only in subjects with high triglycerides [4]. CETP concentration [5] and CETP activity [6] have also shown a positive correlation with increasing carotid intima media thickness as a surrogate endpoint for CAD.

CETP regulates the exchange of cholesteryl esters (CE) and triglycerides (TG) between the apolipoprotein B (apoB)-containing lipoproteins and HDL in plasma. Accordingly, the role that CETP plays in atherosclerosis is complex and may depend on several factors, including the plasma concentration of CETP, the plasma levels and composition (lipids and lipoproteins) of lipoprotein donors (HDL) as well as acceptors (apoB-containing lipoproteins), and the overall metabolic condition [7]. Pharmacologic intervention may, therefore, be complicated and depend on individual patient characteristics. At the present time, both the level of CETP inhibition and the required increase of HDL-C levels to prevent cardiovascular events in patients remain unknown. In this article we summarize data on CETP in various dyslipidemias. In addition, we address the impact of statins and fibrates on CETP activity and also outline ongoing clinical trials with CETP inhibitors as well as provide a summary of future trials.

\section{CETP in Plasma and Lipid Metabolism}

CETP is a hydrophobic glycoprotein that is produced in the liver and adipose tissue. In the plasma compartment, the majority of CETP is bound to HDL, whereas only $1 \%$ of plasma CETP is present in free form [8]. The CETP concentration in healthy subjects varies between 1 and $3 \mu \mathrm{g} / \mathrm{mL}$. In subjects with dyslipidemia, CETP concentra-

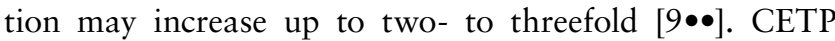
promotes the transfer of CEs and triglycerides between HDL and apoB-containing plasma lipoproteins. HDL is the 


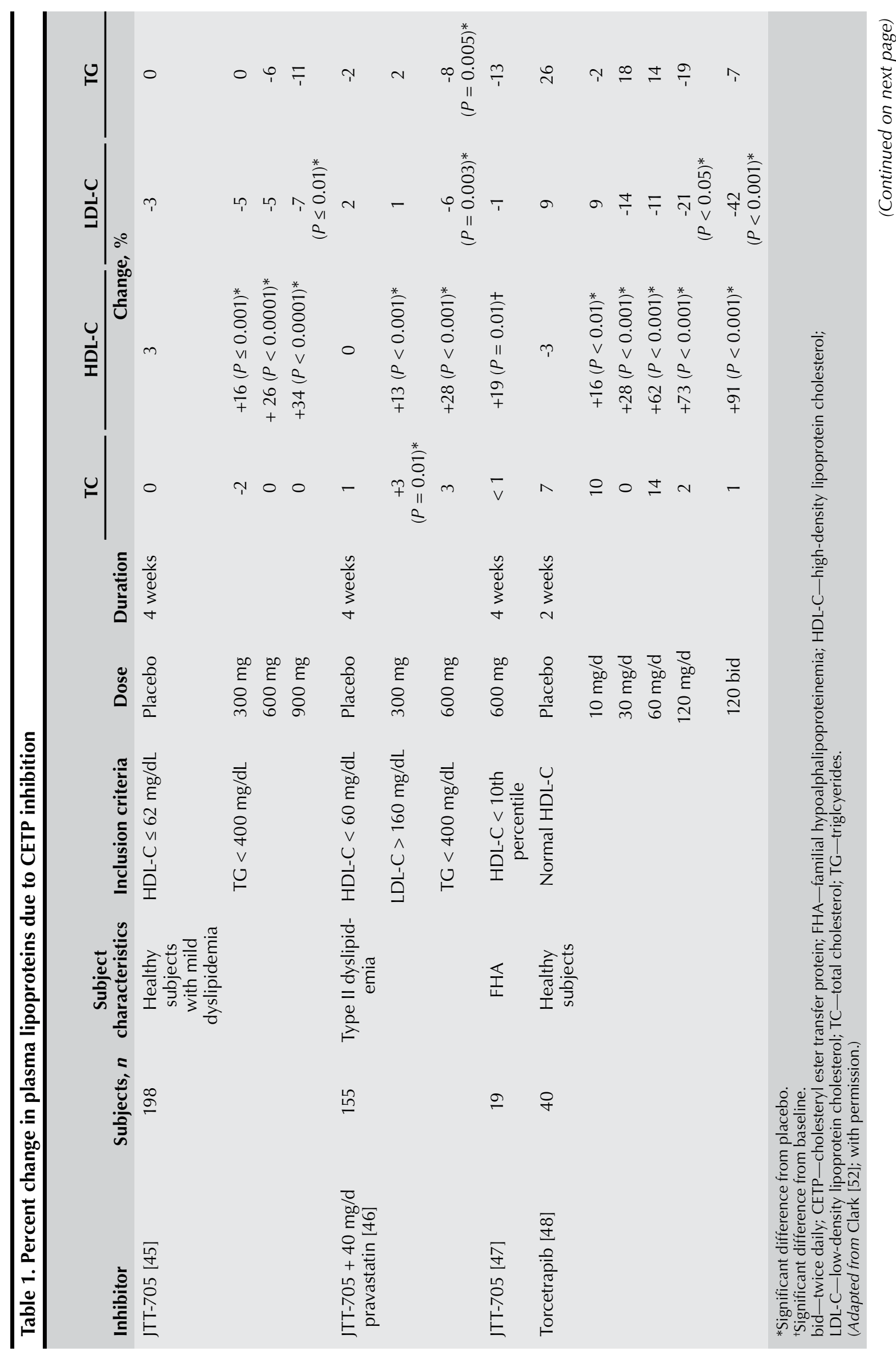




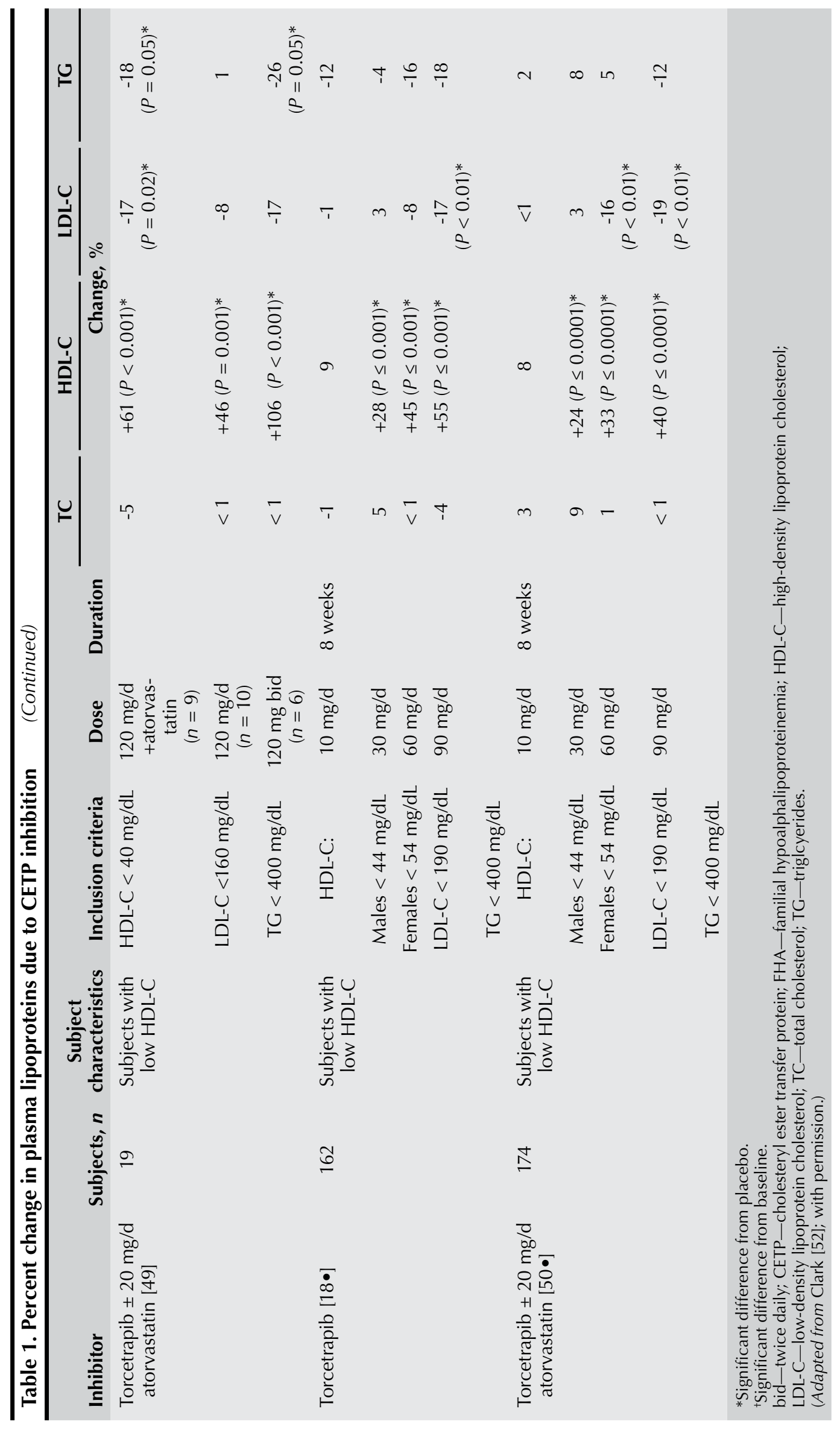


primary lipoprotein particle on which CEs are generated by the reaction catalyzed by lecithin cholesterol acyltransferase (LCAT). TG, however, enter the plasma as a part of triglyceride-rich lipoproteins (TRLs), which are chylomicrons, and as very low-density lipoproteins (VLDL). The overall effect of CETP is a net mass transfer of CE from HDL to TRLs in exchange for TG from TRLs to low-density lipoprotein (LDL) and HDL [10]. The rate of this reaction is not only determined by the amount of CETP in plasma, but also by the concentration and composition of its $\mathrm{CE}$ donor (predominantly HDL) and acceptor (TRL) [11]

\section{CETP in Hypertriglyceridemia}

CETP activity is increased in hypertriglyceridemic patients [12] but not necessarily associated with an increase in CETP mass [13]. As a consequence of the high concentration of TRLs, the TG exchange surplus by CETP will start using LDL on top of HDL as a suitable CE donor. As a consequence, CETP activity results in a shift towards TG-enriched LDL and HDL particles, which both are substrates for hepatic lipase, leading to the formation of small, dense LDL particles [10] as well as smaller HDL particles [14]. The latter changes have been associated with a proatherogenic state. In fact, CETP has been shown to predict CAD predominantly in patients with high triglyceride levels [4]. These findings suggest that CETP inhibition may have beneficial effects in hypertriglyceridemic subjects, but this issue remains to be addressed.

A common form of hyperlipidemia characterized by high TG levels and low HDL-C levels is familial combined hyperlipidemia. This dyslipidemia is characterized by overproduction of VLDL with or without impaired clearance of TRLs [15]. Compared with normolipidemic individuals, patients with combined hyperlipidemia have an increased CETP activity [16], although this finding has not been equivocal [17]. The combination of high triglycerides with low HDL theoretically provides an attractive option for CETP inhibition. However, it should be kept in mind that the impact of CETP inhibition in a situation of long residence time for TRLs needs further evaluation. Recent subanalyses from a trial using torcetrapib [18 $\bullet$ showed that monotherapy with this drug has no effect on triglycerides levels. Surprisingly, in subjects with increased baseline triglycerides, the favorable effect of torcetrapib on LDL-C disappeared altogether. Hence, the exact efficacy of CETP inhibition in familial combined hyperlipidemia needs further study. The Rating Atherosclerotic Disease change by Imaging with A New CETP inhibitor (RADIANCE) study is designed to test the effect of the CETP inhibitor torcetrapib in patients with mixed hyperlipidemia (LDL-C > $160 \mathrm{mg} / \mathrm{dL}$ and triglycerides $>150 \mathrm{mg} / \mathrm{dL}$ ). This study will provide further insight in the effect of CETP inhibition in hypertriglyceridemic states.

\section{Metabolic Disorders and CETP}

Adipose tissue is the second major source of CETP production. As a consequence, plasma CETP concentration closely correlates with the amount of adipose tissue [19]. It has been shown that both CETP mass and activity are increased in obese subjects as compared with non-obese individuals [19]. Weight reduction has been shown to reduce CETP mass and activity in morbidly obese women [20]. Obesity, and especially abdominal obesity, is part of the metabolic syndrome (MS), a condition with increasing prevalence worldwide. MS, defined as abdominal obesity, hypertension, insulin resistance, and dyslipidemia, is characterized by low HDL-C and high triglycerides. Plasma CETP levels were higher in individuals with MS compared with subjects without MS, but only in men [21]. In addition, plasma CETP levels increased with increasing number of components of MS and correlated negatively with LDL size [21]. In this metabolic setting with increased adipose tissue and increased CETP activity, CETP increases the exchange of TG from VLDL particles to HDL particles, which are cleared more quickly by the kidney, resulting in lower HDL-C levels. CETP may also accelerate the exchange of TG (for cholesterol) from VLDL to LDL particles, resulting in more triglyceride-rich LDL particles.

Diabetes mellitus (DM) is a strong risk factor for the development of premature atherosclerosis. Patients with DM generally develop dyslipidemia that is characterized by low HDL-C and high TG levels, both risk factors for atherosclerosis in DM [22]. Small, dense LDL and increased VLDL secretion are also recognized as key features of diabetic dyslipidemia. The level of CETP activity in diabetes yields conflicting results depending on how the measurement was performed (eg, exogenous or endogenous) [22].

In type 2 DM, CETP activity was reported increased, not altered or reduced $[9 \bullet \bullet$. Glycation of lipoproteins that accept CE from HDL has been reported to be responsible for the increased plasma CETP activity in type $2 \mathrm{DM}$ [23]. Together with the plasma TG concentration, plasma CETP activity is a determinant of small, dense LDL in type 2 DM $[9 \bullet \bullet, 24]$. These diseases share an important common characteristic, specifically the atherogenic lipid profile, which includes the triad of low HDL-C, high TG, and increased small, dense LDL particles. In this context the results of RADIANCE II are of major importance because most of the disorders with low HDL-C share the same atherogenic profile. The success of this trial may extend the clinical use of CETP inhibitors in other diseases that are characterized by low HDL-C and high TG, such as diabetes and MS.

\section{CETP and Familial Hypercholesterolemia}

Familial hypercholesterolemia $(\mathrm{FH})$ is a hereditary disorder characterized by elevated levels of plasma LDL and premature cardiovascular disease (CVD). Whereas LDL-C is the primary therapy target, low HDL-C was found to constitute 
an independent and strong risk factor for the development of CVD in FH patients [25]. Both plasma CETP concentration [5,26] and CETP activity [26] were demonstrated to be increased in individuals with hypercholesterolemia, and these patients show increased transfer of CE from HDL to apoB-containing lipoproteins, specifically to dense

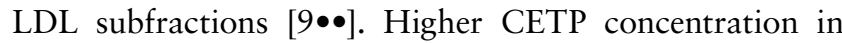
$\mathrm{FH}$ is correlated with higher LDL-C and apoB levels, a higher number of LDL particles, and smaller LDL size [5]. Therefore, CETP appears to be proatherogenic in FH by increasing the cholesterol content in apoB-containing lipoproteins that are known to accumulate in these patients. At present it is unknown how FH patients respond to CETP inhibition. RADIANCE I is a study designed to assess the effects of the CETP inhibitor torcetrapib on atherosclerotic progression in patients with heterozygous $\mathrm{FH}$. Results of this study are anticipated in 2007.

\section{Environmental and Lifestyle Factors Affecting CETP}

Plasma CETP is affected by variety of metabolic conditions that are in themselves associated with changes in CAD risk. It has been shown that alcohol intake and physical exercise are associated with decreased CETP concentration $[27,28]$ and that smoking is associated with high CETP activity [29]. In postmenopausal women, the lipoprotein profile is shifted towards a more atherogenic profile that is improved by hormone replacement therapy [30]. Compared with premenopausal women, CETP activity in postmenopausal women is significantly increased [30]. However, hormone replacement therapy does not appear to affect either plasma CETP levels or activity in postmenopausal women $[9 \bullet \bullet, 30]$.

\section{Pharmacologic Modulation of CETP in Humans}

\section{Trials examining the impact of statins on CETP}

Statins have been proven to reduce the risk of cardiovascular events by lowering the plasma levels of total and LDL-C. Statins have also been shown to decrease CETP activity through lowering plasma CETP concentration and by reducing the number of apoB-containing lipoprotein particles $[9 \bullet \bullet]$. In normolipidemic subjects, simvastatin has been shown to reduce CETP concentration and activity after 6 weeks of treatment [31]. A dose-dependent decrease in CETP activity with simvastatin was observed in normotriglyceridemic subjects with premature CAD [32], although HDL-C remained unchanged. Atorvastatin has been shown to lower CETP activity in hypertriglyceridemic subjects [33] in a dosedependent fashion. In combined hyperlipidemic subjects [34] and in patients with types IIa and IIb primary hyperlipoproteinemia [35], atorvastatin also produced a significant decrease in CETP activity. Whereas in the latter study HDL-C levels were not changed, increases in paraoxonase activity were observed, suggesting improvement of the anti-inflammatory capacity of HDL-C [35]. In FH, pravastatin decreases CETP activity without affecting plasma CETP concentration [36]. Pravastatin reduced the elevated flux of CE from HDL to apoB-containing lipoproteins in these subjects as a result of a reduction in the LDL particle acceptor concentration [36]. Finally, in patients with type 2 diabetes mellitus, pravastatin demonstrated a decrease in CETP activity after 8 weeks of treatment [37]. In a study with pravastatin in CAD subjects, higher CETP concentration was associated with faster progression of coronary atherosclerosis after 2 years of treatment [38]. Similarly, subjects with the highest baseline CETP levels showed the highest improvement in lipoprotein profile and angiographic parameters after 2 years of treatment with pravastatin. In a prospective longitudinal observational study with pravastatin, patients with high plasma CETP concentrations at baseline were associated with fewer cardiovascular events compared with low plasma CETP concentrations in CAD patients treated with pravastatin [39].

\section{Trials examining the impact of fibrates on CETP}

Fibrates are peroxisome proliferator-activated receptor $\alpha(\operatorname{PPAR} \alpha)$ ligands of moderate binding affinity that increase HDL-C levels by up to $15 \%$ [2]. Fibrates are currently used in the treatment of hypertriglyceridemia in the presence or absence of low HDL-C. Fibrates decrease triglyceride-rich acceptor lipoproteins, which can be expected to contribute to reduced plasma CETP activity. However, the effect of fibrates on CETP activity is variable. Some fibrates reduce both CETP concentration and mass, whereas CETP activity has also been reported to increase. In a study evaluating two fibrates (gemfibrozil and bezafibrate), plasma CETP concentration remained unaltered in patients with type IIb hypercholesterolemia [40]. Despite high CETP activity in this population, neither gemfibrozil nor bezafibrate had an effect on CETP activity, although the decrease compared with placebo was borderline significant for bezafibrate. It has been suggested that the effect of bezafibrate on CETP activity is mediated by the decrease and the compositional change of the TRLs, which are the acceptors for CEs from HDL [40]. Fenofibrate decreases CETP activity in subjects with combined hyperlipidemia [41] and in patients with MS [42]. Gemfibrozil also decreases CETP activity in type IIa hyperlipidemic patients [43] but not in normolipidemic subjects [44].

\section{Trials with CETP inhibitors}

Two pharmacologic small-molecule inhibitors of CETP, JTT-705 and torcetrapib, are in an advanced phase of investigation and have been shown to successfully raise HDL cholesterol in humans (Table 1). 
JTT-705

JTT-705 inhibits CETP activity by forming a disulfide bond that causes irreversible binding to the protein. In a randomized, double-blind, placebo-controlled trial of 198 healthy individuals with mild dyslipidemia, JTT-705 was evaluated at doses of 300,600 , and $900 \mathrm{mg} / \mathrm{d}$ for 4 weeks [45]. In the highest dose, a decrease in CETP activity of $37 \%$ was measured concomitantly with a $34 \%$ increase in HDL-C from baseline after 4 weeks of treatment. At this dose, LDL-C was decreased by $7 \%$ and levels of TG were unaffected.

JTT-705 in combination with pravastatin has been investigated in a randomized, double-blind, placebo-controlled trial conducted in 155 individuals with elevated LDL-C who were already taking $40 \mathrm{mg} / \mathrm{d}$ of pravastatin [46]. After 4 weeks, JTT-705, $600 \mathrm{mg} / \mathrm{d}$ plus pravastatin decreased CETP activity by $30 \%$ and increased HDLC by $28 \%$ from baseline, whereas LDL-C decreased by $6 \%$ and TG by $8 \%$ from baseline. JTT-705, $300 \mathrm{mg}$ plus pravastatin was about half as effective as the higher dose, decreasing CETP activity by approximately $16 \%$ and increasing HDL-C by approximately $13 \%$ [46]. This dose did not influence LDL or TG levels. The combination therapy of JTT-705 with pravastatin was well tolerated and did not induce significant adverse effects.

In a single-center, randomized, double-blind clinical trial in 19 patients with familial hypoalphalipoproteinemia, 4 weeks of treatment with 600 mg of JTT-705 was associated with a $24 \%$ decrease in CETP activity and a $19 \%$ increase in HDL-C levels [47]. Furthermore, CETP inhibition in this study was associated with favorable effects on HDL and LDL subfractions, with a concomitant reduction of oxidized LDL autoantibodies and enhanced serum paraoxonase activity. Like JTT-705, JTT-302 is an orally administered CETP inhibitor and is currently undergoing phase I trials for the potential treatment of dyslipidemia.

\section{Torcetrapib}

Torcetrapib is a potent and selective inhibitor of CETP. It enhances the association between CETP and HDL, forming a complex that inhibits the transfer of lipids between HDL and other lipoproteins. In the first study with torcetrapib, 40 healthy normolipidemic subjects were randomized to receive placebo or increasing doses of torcetrapib ranging from 10 to $240 \mathrm{mg} / \mathrm{d}$ for 14 days. Activity of CETP was reduced by $12 \%$ to $80 \%$. The concentration of HDL-C increased by $16 \%$ to $91 \%$ and that of LDL-C decreased by $21 \%$ to $42 \%$ [48]. In a second study, 19 subjects with low HDL-C levels $(<38.7 \mathrm{mg} / \mathrm{dL})$ were treated with torcetrapib at doses up to $120 \mathrm{mg}$ twice daily [49]. Some subjects also received atorvastatin. Torcetrapib $(120 \mathrm{mg})$ increased HDL-C by $61 \%$ and $46 \%$ in the presence and absence of atorvastatin, respectively. In six subjects who received $120 \mathrm{mg}$ twice daily for another 4 weeks, torcetrapib increased HDL-C by $106 \%$. Further- more, a $17 \%$ reduction in LDL-C levels was observed in the atorvastatin group. Both LDL and HDL particle size increased with torcetrapib treatment.

Recently, the efficacy and safety of torcetrapib monotherapy in combination with atorvastatin was investigated in two studies with patients with below-average HDL-C levels (men $<44 \mathrm{mg} / \mathrm{dL}$ and women $<54 \mathrm{mg} / \mathrm{dL}$ ). In the first study, 162 patients who did not receive lipid-lowering therapy were randomized to receive increasing doses of torcetrapib $(10-90 \mathrm{mg} / \mathrm{d})$ [18•]. After 8 weeks of treatment a dose-dependent increase in HDL-C levels was observed, with a maximum increase of $55 \%$ in the highest-dose group $(90 \mathrm{mg} / \mathrm{d})$. This increase was accompanied by a $23 \%$ increase in apoA-I levels and a shift to larger parts of both HDL and LDL particles. In the highest-dose group, LDL-C levels decreased by $17 \%$; however, this LDL-C-lowering effect was completely lost in patients with high baseline TG levels, suggesting that CETP inhibition may be insufficient as a monotherapy in patients with high TG levels. It was suggested that in a situation of high TG, the combination of compositional changes in VLDL and CETP inhibition leads to accelerated conversion of VLDL to LDL via lipoprotein lipases, which may abolish the effect of torcetrapib on LDL-C levels. Although there were no serious adverse effects, significant blood pressure increases were noted in two of the 140 subjects.

In the second study, 174 subjects who were already taking $20 \mathrm{mg} / \mathrm{d}$ of atorvastatin were randomized to 8 weeks of double-blind treatment with placebo or increasing doses of torcetrapib [50•]. In general, the changes in the levels of HDL-C, LDL-C, and their respective apolipoproteins and particle sizes in this study were similar to that in the monotherapy study. However, the percent increase from baseline in HDL-C was less marked in the atorvastatin/torcetrapib group compared with the monotherapy group. For example, $90 \mathrm{mg} / \mathrm{d}$ of torcetrapib caused a $55 \%$ increase in HDL-C levels in the monotherapy study whereas the same dose yielded a $40 \%$ increase in the atorvastatin/torcetrapib group. This difference may lie in the fact that atorvastatin decreases CETP activity and in that way additional inhibition of CETP by torcetrapib yields less HDL-C increase. There were significant decreases in LDL-C levels in the two highest groups (60 and $90 \mathrm{mg} / \mathrm{d}$ ) of $16 \%$ and $19 \%$, respectively, and these effects were independent of baseline TG levels. Statin therapy may help in reducing accumulation of LDL-C in patients with hypertriglyceridemia by upregulation of LDL receptors. In this study $2.9 \%$ of the subjects showed a significant increase in blood pressure levels. The potential clinical relevance of this adverse effect is currently being evaluated.

\section{CETP Inhibition and (Surrogate) Endpoints}

The current CETP inhibitors JTT-705 and torcetrapib have proven to be efficacious in increasing HDL-C levels 
both when used as monotherapy or in combination with statin therapy. Moreover, they increase the mean size of HDL and LDL particles, an effect on the lipid profile which is similar with previous reports of large HDL particles in individuals with CETP deficiency. Recently, Matsuura et al. [51•] have shown that HDL from CETPdeficient subjects shows enhanced ability to promote cholesterol efflux from macrophages, which is an important step in the reverse cholesterol transport pathway. The key question is, however, whether these compounds will be able to reduce cardiovascular events. Two studies on surrogate and clinical endpoints are ongoing with torcetrapib. The Investigation of Lipid Level management Using Coronary Ultrasound to Assess Reduction of Atherosclerosis by CETP Inhibition and HDL Elevation (ILLUSTRATE) study is evaluating changes in atheroma volume in coronary arteries using intravascular ultrasound. A total of 1191 patients with CHD have been randomized to treatment, with results expected in the first quarter of 2007. A large clinical endpoint study with torcetrapib, the Investigation of Lipid Level Management to Understand Its Impact in Atherosclerotic Events (ILLUMINATE) study, has also been initiated. This study has randomized approximately 15,000 subjects with coronary heart disease or coronary heart diseaserisk equivalents to receive either torcetrapib/atorvastatin or atorvastatin alone. The primary endpoint is composite of death, nonfatal myocardial infarction, or stroke. The most recent development is the premature discontinuation of this trial on December 2, 2006 due to increased mortality rate in the torcetrapib-treated subjects. Thus, the combination of atorvastatin plus torcetrapib was linked to a $60 \%$ higher mortality rate compared with atorvastatin alone (ie, 82 deaths in the torcetrapib/atorvastatin group versus 51 deaths in the atorvastatin group). Of note, at present it is unclear whether the increase in cardiovascular mortality only applies to the torcetrapib compound or to all CETP inhibitors. In support of the former, torcetrapib has been linked to onset of hypertension, whereas other CETP inhibitors do not show this particular side effect.

\section{Conclusions}

The relationship between CETP and lipoprotein metabolism is complex and may differ in various metabolic settings. CETP inhibitors exert a wide array of beneficial effects on the lipid profile, including an increase in HDL and a decrease in the number of small, dense LDL particles. Until recently, safety and efficacy data on CETP inhibitors were good. The premature cessation of the endpoint trial using CETP inhibitor torcetrapib (ILLUMINATE), showing higher mortality during active treatment, has further complicated the matter. Future research has to unravel whether this adverse effect is com- pound specific (eg, due to hypertension) or whether it will prove to be a complication for all CETP inhibitors.

\section{References and Recommended Reading}

Papers of particular interest, published recently, have been highlighted as:

- Of importance

-• Of major importance

1. Doggrell SA: Inhibitors of cholesteryl ester transfer protein-a new approach to coronary artery disease. Exp Opin Invest Drugs 2006, 15:99-106.

2. Birjmohun RS, Hutten BA, Kastelein JJ, Stroes ES: Efficacy and safety of high-density lipoprotein cholesterol-increasing compounds: a meta-analysis of randomized controlled trials. J Am Coll Cardiol 2005, 45:185-197.

3. Curb JD, Abbott RD, Rodriguez BL, et al.: A prospective study of HDL-C and cholesteryl ester transfer protein gene mutations and the risk of coronary heart disease in the elderly. J Lipid Res 2004, 45:948-953.

4. Boekholdt SM, Kuivenhoven JA, Wareham NJ, et al.: Plasma levels of cholesteryl ester transfer protein and the risk of future coronary artery disease in apparently healthy men and women: the Prospective EPIC (European Prospective Investigation into Cancer and nutrition)-Norfolk Population Study. Circulation 2004, 110:1418-1423.

5. de Grooth GJ, Smilde TJ, van Wissen S, et al.: The relationship between cholesteryl ester transfer protein levels and risk factor profile in patients with familial hypercholesterolemia. Atherosclerosis 2004, 173:261-267.

6. de Vries R, Perton FG, Linga-Thie GM, et al.: Plasma cholesteryl ester transfer is a determinant of intima-media thickness in type 2 diabetic and nondiabetic subjects: role of cetp and triglycerides. Diabetes 2005, 54:3554-3559.

7. Sikorski JA: Oral cholesteryl ester transfer protein (CETP) inhibitors: a potential new approach for treating coronary artery disease. J Med Chem 2006, 49:1-22.

8. Nishida HI, Arai H, Nishida T: Cholesterol ester transfer mediated by lipid transfer protein as influenced by changes in the charge characteristics of plasma lipoproteins. J Biol Chem 1993, 268:16352-16360.

9.• Le Goff W, Guerin M, Chapman MJ: Pharmacological modulation of cholesteryl ester transfer protein, a new therapeutic target in atherogenic dyslipidemia. Pharmacol Ther 2004, 101:17-38. This is an overview on many biochemical details of CETP. It also contains a detailed summary of data on CETP inhibition in animals. 10. Barter PJ, Brewer HB Jr, Chapman MJ, et al.: Cholesteryl ester transfer protein: a novel target for raising HDL and inhibiting atherosclerosis. Arterioscler Thromb Vasc Biol 2003, 23:160-167.

11. Borggreve SE, de Vries R, Dullaart RP: Alterations in high-density lipoprotein metabolism and reverse cholesterol transport in insulin resistance and type 2 diabetes mellitus: role of lipolytic enzymes, lecithin:cholesterol acyltransferase and lipid transfer proteins. Eur J Clin Invest 2003, 33:1051-1069.

12. Tato F, Vega GL, Grundy SM: Determinants of plasma HDL-cholesterol in hypertriglyceridemic patients: role of cholesterol-ester transfer protein and lecithin cholesteryl acyl transferase. Arterioscler Thromb Vasc Biol 1997, 17:56-63.

13. Mann CJ, Yen FT, Grant AM, Bihain BE: Mechanism of plasma cholesteryl ester transfer in hypertriglyceridemia. J Clin Invest 1991, 88:2059-2066.

14. Kontush A, Chapman MJ: Antiatherogenic small, dense HDL-guardian angel of the arterial wall? Nat Clin Pract Cardiovasc Med 2006, 3:144-153. 
15. Venkatesan S, Cullen P, Pacy P, et al.: Stable isotopes show a direct relation between VLDL apoB overproduction and serum triglyceride levels and indicate a metabolically and biochemically coherent basis for familial combined hyperlipidemia. Arterioscler Thromb 1993, 13:1110-1118.

16. Tato F, Vega GL, Tall AR, Grundy SM: Relation between cholesterol ester transfer protein activities and lipoprotein cholesterol in patients with hypercholesterolemia and combined hyperlipidemia. Arterioscler Thromb Vasc Biol 1995, 15:112-120.

17. Vakkilainen J, Jauhiainen M, Ylitalo K, et al.: LDL particle size in familial combined hyperlipidemia: effects of serum lipids, lipoprotein-modifying enzymes, and lipid transfer proteins. J Lipid Res 2002, 43:598-603.

18. Davidson MH, McKenney JM, Shear CL, Revkin JH: Efficacy and safety of torcetrapib, a novel cholesteryl ester transfer protein inhibitor, in individuals with below-average high-density lipoprotein cholesterol levels. J Am Coll Cardiol 2006, 48:1774-1781.

This is a study documenting the safety and efficacy of torcetrapib in patients with low HDL-C levels.

19. Arai T, Yamashita S, Hirano K, et al.: Increased plasma cholesteryl ester transfer protein in obese subjects. A possible mechanism for the reduction of serum HDL cholesterol levels in obesity. Arterioscler Thromb 1994, 14:1129-1136.

20. Ebenbichler CF, Laimer M, Kaser S, et al.: Relationship between cholesteryl ester transfer protein and atherogenic lipoprotein profile in morbidly obese women. Arterioscler Thromb Vasc Biol 2002, 22:1465-1469.

21. Sandhofer A, Kaser S, Ritsch A, et al.: Cholesteryl ester transfer protein in metabolic syndrome. Obesity 2006, 14:812-818.

22. Quintao EC, Medina WL, Passarelli M: Reverse cholesterol transport in diabetes mellitus. Diabetes Metab Res Rev 2000, 16:237-250.

23. Passarelli M, Catanozi S, Nakandakare ER, et al.: Plasma lipoproteins from patients with poorly controlled diabetes mellitus and "in vitro" glycation of lipoproteins enhance the transfer rate of cholesteryl ester from HDL to apo-Bcontaining lipoproteins. Diabetologia 1997, 40:1085-1093.

24. Lahdenpera S, Syvanne M, Kahri J, Taskinen MR: Regulation of low-density lipoprotein particle size distribution in NIDDM and coronary disease: importance of serum triglycerides. Diabetologia 1996, 39:453-461.

25. Jansen AC, van Aalst-Cohen ES, Tanck MW, et al.: The contribution of classical risk factors to cardiovascular disease in familial hypercholesterolaemia: data in 2400 patients. J Intern Med 2004, 256:482-490.

26. Bagdade JD, Ritter MC, Subbaiah PV: Accelerated cholesteryl ester transfer in plasma of patients with hypercholesterolemia. J Clin Invest 1991, 87:1259-1265.

27. Hannuksela M, Marcel YL, Kesaniemi YA, Savolainen MJ: Reduction in the concentration and activity of plasma cholesteryl ester transfer protein by alcohol. J Lipid Res 1992, 33:737-744.

28. Seip RL, Moulin P, Cocke T, et al.: Exercise training decreases plasma cholesteryl ester transfer protein. Arterioscler Thromb 1993, 13:1359-1367.

29. Dullaart RP, Hoogenberg K, Dikkeschei BD, van TA: Higher plasma lipid transfer protein activities and unfavorable lipoprotein changes in cigarette-smoking men. Arterioscler Thromb 1994, 14:1581-1585.

30. Lewis-Barned NJ, Sutherland WH, Walker RJ, et al.: Plasma cholesterol esterification and transfer, the menopause, and hormone replacement therapy in women. J Clin Endocrinol Metab 1999, 84:3534-3538.

31. Ahnadi CE, Berthezene F, Ponsin G: Simvastatin-induced decrease in the transfer of cholesterol esters from high density lipoproteins to very low and low density lipoproteins in normolipidemic subjects. Atherosclerosis 1993, 99:219-228.
32. van Wijk JP, Buirma R, van Tol A, et al.: Effects of increasing doses of simvastatin on fasting lipoprotein subfractions, and the effect of high-dose simvastatin on postprandial chylomicron remnant clearance in normotriglyceridemic patients with premature coronary sclerosis. Atherosclerosis 2005, 178:147-155.

33. Le NA, Innis-Whitehouse W, Li X, et al.: Lipid and apolipoprotein levels and distribution in patients with hypertriglyceridemia: effect of triglyceride reductions with atorvastatin. Metabolism 2000, 49:167-177.

34. Guerin M, Lassel TS, Le Goff W, et al.: Action of atorvastatin in combined hyperlipidemia: preferential reduction of cholesteryl ester transfer from HDL to VLDL1 particles. Arterioscler Thromb Vasc Biol 2000, 20:189-197.

35. Kassai A, Illyes L, Mirdamadi HZ, et al.: The effect of atorvastatin therapy on lecithin:cholesterol acyltransferase, cholesteryl ester transfer protein and the antioxidant paraoxonase. Clin Biochem 2006, In press.

36. Guerin M, Dolphin PJ, Talussot C, et al.: Pravastatin modulates cholesteryl ester transfer from hdl to apob-containing lipoproteins and lipoprotein subspecies profile in familial hypercholesterolemia. Arterioscler Thromb Vasc Biol 1995, 15:1359-1368.

37. Bhatnagar D, Durrington PN, Kumar S, et al.: Effect of treatment with a hydroxymethylglutaryl coenzyme A reductase inhibitor on fasting and postprandial plasma lipoproteins and cholesteryl ester transfer activity in patients with NIDDM. Diabetes 1995, 44:460-465.

38. Klerkx AH, de Grooth GJ, Zwinderman AH, et al.: Cholesteryl ester transfer protein concentration is associated with progression of atherosclerosis and response to pravastatin in men with coronary artery disease (REGRESS). Eur J Clin Invest 2004, 34:21-28.

39. Marschang P, Sandhofer A, Ritsch A, et al.: Plasma cholesteryl ester transfer protein concentrations predict cardiovascular events in patients with coronary artery disease treated with pravastatin. J Intern Med 2006, 260:151-159.

40. Durrington PN, Mackness MI, Bhatnagar D, et al.: Effects of two different fibric acid derivatives on lipoproteins, cholesteryl ester transfer, fibrinogen, plasminogen activator inhibitor and paraoxonase activity in type IIb hyperlipoproteinaemia. Atherosclerosis 1998, 138:217-225.

41. Guerin M, Bruckert E, Dolphin PJ, et al.: Fenofibrate reduces plasma cholesteryl ester transfer from HDL to VLDL and normalizes the atherogenic, dense LDL Profile in combined hyperlipidemia. Arterioscler Thromb Vasc Biol 1996, 16:763-772.

42. Watts GF, Ji J, Chan DC, et al.: Relationships between changes in plasma lipid transfer proteins and apolipoprotein B-100 kinetics during fenofibrate treatment in the metabolic syndrome. Clin Sci (London) 2006, 111:193-199.

43. Franceschini G, Lovati MR, Manzoni C, et al.: Effect of gemfibrozil treatment in hypercholesterolemia on low density lipoprotein (LDL) subclass distribution and LDL-cell interaction. Atherosclerosis 1995, 114:61-71.

44. Ponsin G, Girardot G, Berthezene F: Mechanism of the gemfibrozil-induced decrease in the transfer of cholesterol esters from high density lipoproteins to very low and low density lipoproteins. Biochem Med Metab Biol 1994, 52:58-64.

45. de Grooth GJ, Kuivenhoven JA, Stalenhoef AF, et al.: Efficacy and safety of a novel cholesteryl ester transfer protein inhibitor, JTT-705, in humans: a randomized phase II dose-response study. Circulation 2002, 105:2159-2165.

46. Kuivenhoven JA, de Grooth GJ, Kawamura H, et al.: Effectiveness of inhibition of cholesteryl ester transfer protein by JTT-705 in combination with pravastatin in type II dyslipidemia. Am J Cardiol 2005, 95:1085-1088.

47. Bisoendial RJ, Hovingh GK, El HK, et al.: Consequences of cholesteryl ester transfer protein inhibition in patients with familial hypoalphalipoproteinemia. Arterioscler Thromb Vasc Biol 2005, 25:e133-e134. 
48. Clark RW, Sutfin TA, Ruggeri RB, et al.: Raising high-density lipoprotein in humans through inhibition of cholesteryl ester transfer protein: an initial multidose study of torcetrapib. Arterioscler Thromb Vasc Biol 2004, 24:490-497.

49. Brousseau ME, Schaefer EJ, Wolfe ML, et al.: Effects of an inhibitor of cholesteryl ester transfer protein on HDL cholesterol. N Engl J Med 2004, 350:1505-1515.

50. McKenney JM, Davidson MH, Shear CL, Revkin JH: Efficacy and safety of torcetrapib, a novel cholesteryl ester transfer protein inhibitor, in individuals with below-average high-density lipoprotein cholesterol levels on a background of atorvastatin. J Am Coll Cardiol 2006, 48:1782-1790.

This is a study documenting the safety and efficacy of torcetrapib in patients with low HDL-C levels on a backround of atorvastatin.
51. Matsuura F, Wang N, Chen W, et al.: HDL from CETP-deficient subjects shows enhanced ability to promote cholesterol efflux from macrophages in an apoE- and ABCG1-dependent pathway. J Clin Invest 2006, 116:1435-1442.

This study shows that HDL from CETP-deficient subjects is able to promote cholesterol efflux from macrophages. 